\title{
Application of a New Sensing Principle for Photoacoustic Imaging of Point Absorbers
}

\author{
Zafer Doğan ${ }^{a, b}$, Ivana Jovanovic ${ }^{a, b}$, Thierry Blu $^{c}$, Dimitri Van De Ville ${ }^{a, b}$ \\ ${ }^{a}$ École Polytechnique Fédérale de Lausanne, Switzerland \\ ${ }^{b}$ University of Geneva, Switzerland \\ ${ }^{c}$ The Chinese University of Hong Kong, China
}

\begin{abstract}
Photoacoustic tomography (PAT) is a hybrid imaging method, which combines ultrasonic and optical imaging modalities, in order to overcome their respective weaknesses and to combine their strengths. It is based on the reconstruction of optical absorption properties of the tissue from the measurements of a photoacousticallygenerated pressure field. Current methods consider laser excitation, under thermal and stress confinement assumptions, which leads to the generation of a propagating pressure field. Conventional reconstruction techniques then recover the initial pressure field based on the boundary measurements by iterative reconstruction algorithms in time- or Fourier-domain. Here, we propose an application of a new sensing principle that allows for efficient and non-iterative reconstruction algorithm for imaging point absorbers in PAT. We consider a closed volume surrounded by a measurement surface in an acoustically homogeneous medium and we aim at recovering the positions and the amount of heat absorbed by these absorbers. We propose a two-step algorithm based on proper choice of so-called sensing functions. Specifically, in the first step, we extract the projected positions on the complex plane and the weights by a sensing function that is well-localized on the same plane. In the second step, we recover the remaining z-location by choosing a proper set of plane waves. We show that the proposed families of sensing functions are sufficient to recover the parameters of the unknown sources without any discretization of the domain. We extend the method for sources that have joint-sparsity; i.e., the absorbers have the same positions for different frequencies. We evaluate the performance of the proposed algorithm using simulated and noisy sensor data and we demonstrate the improvement obtained by exploiting joint sparsity.
\end{abstract}

Keywords: photoacoustic imaging, sensing principle, source imaging, k-space models, inverse problem, source localization, wave equation, Helmholtz equation, joint sparsity

\section{INTRODUCTION}

Photoacoustic tomograpy (PAT) is a hybrid imaging modality that combines high intrinsic contrast of optical imaging and high spatial resolution of ultrasonic imaging. It is based on the generation of acoustic waves due to the tissue dependent absorption of optical energy. The absorbed energy initially creates a thermally induced pressure jump that leads to an emergence of the propagating acoustic waves in the tissue. ${ }^{1,2}$ Then the activated field is recorded with ultrasound sensors placed on the surface of the volume. The problem is to recover the absorption properties of the tissue from the measured data.

Numerous approaches have been proposed for the inverse problem of PAT. Many of these algorithms are based on the inversion of the generalized spherical Radon transform and requires the measured data to be densely sampled..$^{3,4}$ Exact analytical solutions in both time and frequency domains are provided assuming continuous aperture with infinite bandwidth. ${ }^{5,6} \mathrm{~A}$ variety of iterative reconstruction algorithms are proposed to solve the problem for lower sampling density, ${ }^{7,8}$ which mostly requires computationally heavy iterations.

In this paper, we propose a new non-iterative reconstruction framework for the detection of point absorbers in PAT based on the measured data that has a certain spatial sampling density. First, we propose the sensing principle to provide a link between the volumetric parameters of the unknown absorption profile and the measured

Further author information: (Send correspondence to Z.D.)

Z.D.: E-mail: zafer.dogan@epfl.ch, Telephone: +41 216930610

Photons Plus Ultrasound: Imaging and Sensing 2013, edited by Alexander A. Oraevsky, Lihong V. Wang, Proc. of SPIE Vol. 8581, 85813Z · (C) 2013 SPIE · CCC code: 1605-7422/13/\$18 - doi: 10.1117/12.2005063 
data. Then, we propose a two-step algorithm based on proper choice of the sensing functions that are solutions of the homogeneous Helmholz equation in the volume. In the first step, we use a sensing function that is welllocalized on the complex plane surrounding the observed volume. Then, we extract the projected positions on the complex plane and the intensity weights of each point source. In the second step, we recover the remaining z-locations by choosing a second set of test function, which are plane waves. Finally, we extend the method for the case when the point absorbers exhibit joint sparsity; i.e., the point absorbers have same positions for different frequencies. We propose to use the data jointly from a frequency range that is known to be less-aliased spatially by the design of the sampling process.

The paper is arranged as follows. In Section 2, we introduce the problem setting of PAT. In Section 3, we provide the main idea of the sensing principle. In Section 4, we develop the the proposed method for a 3D spherical imaging setup. In Section 5, the feasibility of the proposed method is demonstrated with numerical experiments.

\section{PROBLEM SETTING}

In PAT, the optical radiation absorbed by the tissue gives rise to thermal heating. Under the condition of thermal confinement, the tissue subsequently goes into a thermal expansion. The generated acoustic field in which the absorbed energy can be modelled as an heating source is governed by the wave equation

$$
\nabla^{2} p(\mathbf{r}, t)-\frac{1}{c^{2}} \frac{\partial^{2} p(\mathbf{r}, t)}{\partial t^{2}}=-\frac{\beta}{C_{p}} \frac{\partial}{\partial t} H(\mathbf{r}, t)
$$

where $H(\mathbf{r}, t)$ is the heating function that gives rise to an acoustic field $p(\mathbf{r}, t), \beta$ is the isobaric volume expansion coefficient $\left[\mathrm{K}^{-1}\right], C_{p}$ is the specific heat $[\mathrm{J} /(\mathrm{K} \mathrm{kg})]$, and $c$ is the speed of sound. Under the thermal confinement condition, the heating function can be further decomposed as the product

$$
H(\mathbf{r}, t)=A(\mathbf{r}) I(t),
$$

where $A(\mathbf{r})$ is the spatial absorption function and $I(t)$ the temporal illumination function, which is known by the excitation protocol.

In particular, we consider a closed volume of $\Omega$ in free space where the optical absorption occurs with a surface $\partial \Omega$ and the activated acoustic field and its normal derivative are measured on the surface of the volume, i.,e., $\left.p(\mathbf{r}, t)\right|_{\partial \Omega}$ and $\left.\nabla p(\mathbf{r}, t)\right|_{\partial \Omega}$. Then, we parametrize the spatial absorption function

$$
A(\mathbf{r})=\sum_{m=1}^{M} c_{m} \delta\left(\mathbf{r}-\mathbf{r}_{\mathbf{m}}\right),
$$

where $c_{m} \in \mathbb{R}, \mathbf{r}_{m} \in \Omega$. With this parametrization, absorption function is completely characterized by the positions and intensities of $M$ sources, i.e., with $4 \mathrm{M}$ parameters, and the inverse problem becomes well-posed. ${ }^{9}$ The key to the reconstruction problem is to recover the spatial absorption function $A(\mathbf{r})$; i.e., the parameters of (2) from the boundary measurements of the acoustic field.

\section{SENSING PRINCIPLE FOR PAT}

The time harmonic solutions of (1) will be

$$
\nabla^{2} p(\mathbf{r}, \omega)+\frac{\omega^{2}}{c^{2}} p(\mathbf{r}, \omega)=-\frac{\beta}{C_{p}}(i \omega) A(\mathbf{r}) I(\omega),
$$

which is the inhomogeneous Helmholtz equation. Considering a specific frequency, $\omega$, we propose a sensing principle based on the second Green's identity such that we can obtain a link between the absorption function and the measurements on the surface.

$$
\langle\Psi, A\rangle=\alpha(\omega) \oiiint_{\partial \Omega}[\Psi(\mathbf{r}, \omega) \nabla p(\mathbf{r})-p(\mathbf{r}, \omega) \nabla \Psi(\mathbf{r}, \omega)] \cdot \mathbf{e}_{\partial \Omega} d S
$$


where $\alpha(\omega)=i C_{p} /(\beta \omega I(\omega))$ is a constant that we use to compensate the surface integral and $\Psi(\mathbf{r}, \omega)$ is a sensing function satisfying the homogeneous Helmholz equation in the the volume

$$
\nabla^{2} \Psi(\mathbf{r}, \omega)+\frac{\omega^{2}}{c^{2}} \Psi(\mathbf{r}, \omega)=0 \text { in } \Omega .
$$

This way the sensing principle allows to extract volumetric samples of the absorption function through a surface integral of the sensor measurements of the activated acoustic field.

\section{IMAGING OF POINT ABSORBERS}

We propose a two-step algorithm to extract the parameters of the absorption function from the observed acoustic field by means of applying the sensing principle with properly chosen sensing functions.

\subsection{Projection onto Complex Plane}

In the first step, we extract the positions of the point absorbers on the complex plane. For this purpose, we choose a set of test functions $\Psi^{(1)}$ satisfying (5):

$$
\Psi_{n}^{(1)}(\mathbf{r}, \omega)=\frac{e^{i \omega z / c}}{x+i y-a_{n}}, a_{n} \notin \Omega,
$$

where $a_{n}$ 's are the poles of the sensing function on the complex plane located at equidistant angles $a_{n}=a e^{i n \theta}, n \in$ $\llbracket 0, N-1 \rrbracket,|a|$ is greater than the radius of $\Omega$ excluding the volume and $\theta$ is an arbitrary angle. Then, we define a polynomial, $R(X)$ whose roots are the positions of the point absorbers on the XY-plane:

$$
R(X)=\prod_{m=1}^{M}\left(X-s_{m}\right)=\sum_{k=0}^{M} r_{k} X^{k} \text { where } s_{m}=x_{m}+i y_{m} \text { with } r_{M}=1 .
$$

With this selection of the proposed family of sensing function, ${ }^{10}$ the extracted samples of the absorption function (4) turns into an annihilable function as follows:

$$
\begin{aligned}
\left\langle\Psi_{n}^{(1)}, A\right\rangle=\mu_{n} & =\sum_{m=1}^{M} \frac{c_{m} e^{i \omega z_{m} / c}}{x_{m}+i y_{m}-a_{n}} \\
& =\frac{\sum_{m=0}^{M-1} c_{m}^{\prime} e^{i m n \theta}}{\prod_{m=1}^{M}\left(x_{m}+i y_{m}-a_{n}\right)} \\
& =\frac{\sum_{m=0}^{M-1} c_{m}^{\prime} e^{i m n \theta}}{R\left(a_{n}\right)}
\end{aligned}
$$

where $c_{m}^{\prime}$ are complex-valued coefficients that do not depend on $n$ nor $\theta$. The sequence $u=\left\{u_{n}\right\}$ for $n \in \llbracket 0, N-1 \rrbracket$, whose coefficients are defined by $u_{n}=R\left(a_{n}\right) \mu_{n}$, can be annihilated by an FIR digital filter $h=\left\{h_{k}\right\}$ for $k \in$ $\llbracket 0, N-1 \rrbracket$ characterized as follows:

$$
H(z)=\sum_{k \in \mathcal{Z}} h_{k} z^{-k}=\prod_{k=0}^{M-1}\left(1-e^{i k \theta} z^{-1}\right),
$$

where the zeros of the filter are chosen at $e^{i k \theta}$ for $k \in \llbracket 0, M-1 \rrbracket$ to annihilate the sequences $u$ as $h * u=$ $0, \forall n \in \llbracket M, N-1 \rrbracket$. The coefficients of the polynomial $\mathrm{R}(\mathrm{X})$ defined by (7) satisfy the following linear system of equations:

$$
\sum_{k=0}^{M} A_{n, k} r_{k}=0, \quad A_{n, k}=\sum_{n^{\prime}=0}^{N-1} h_{n-n^{\prime}} a_{n^{\prime}}^{k} \mu_{n^{\prime}},
$$

where $\mathrm{n}=\mathrm{M}, \ldots, \mathrm{N}-1$. Finally, solving the linear system expressed as $\mathbf{A} R=0$, the point absorbers' positions on the XY-plane are found to be the roots of the polynomial $R$. 


\section{Determining the weights}

Using the projected locations on XY-plane from the previous section, the same samples in (8) are now used to solve another linear system to extract the weights of the point absorbers. The system will satisfy

$$
\underbrace{\left\langle\Psi_{n}^{(1)}, A\right\rangle=\mu_{n}}_{\text {known }}=\sum_{m=1}^{M} \frac{p_{m}}{\underbrace{x_{m}+i y_{m}}_{\text {projected locs. }}-a_{n}}, p_{m}=c_{m} e^{i \omega z_{m} / c} .
$$

where we redefine $p_{m}$ as a complex variable to be solved. In matrix notation, we can express this system as $\mathcal{B} \mathbf{p}=\mu$, where $\mathcal{B}$ is an $N \times M$ matrix with the entries $\mathcal{B}_{m, n}=\frac{1}{x_{m}+i y_{m}-a_{n}}$, and $\mathbf{p}$ is an $M \times 1$ vector with entries $p_{m}=c_{m} e^{i k z_{m}}$ and $\mu$ is an $N \times 1$ vector with entries $\mu_{n}$.

\subsection{Recovery of the $\mathrm{Z}$ locations}

The phase of the solution in (11) also gives information about the $z$-locations. However, due to the periodicity of the exponential term with $2 \pi c / \omega$, the z-locations cannot be determined with the samples from (8). Previously, we proposed a solution to recover the z-locations using a modified Dijkstra's algorithm ${ }^{11}$ for a point source model that shows joint-sparsity. Here, we propose to recover the remaining z-locations by means of applying the sensing principle with a proper set of plane waves:

$$
\Psi_{n}^{(2)}(\mathbf{r}, \omega)=e^{i\left(\omega_{x} x+\omega_{y} y+\omega_{z} z\right) / c}, \text { s.t. } \omega_{x}^{2}+\omega_{y}^{2}+\omega_{z}^{2}=\omega^{2},
$$

where $\omega_{z}<\frac{\pi c}{R_{o}}, R_{o}$ being the radius of the volume of interest, and $\left(\omega_{x}, \omega_{y}\right)=\sqrt{\omega^{2}-\omega_{z}^{2}}\left(\cos \alpha_{n}, \sin \alpha_{n}\right), \alpha_{n}=$ $\frac{2 \pi}{N} n, n \in \llbracket 0, N-1 \rrbracket$. With this selection of the proposed family of plane waves, the extracted samples of the absorption function become

$$
\begin{aligned}
\left\langle\Psi_{n}^{(2)}, A\right\rangle & =\sum_{m=1}^{M} c_{m} e^{i\left(\omega_{x} x_{m}+\omega_{y} y_{m}+\omega_{z} z_{m}\right) / c} \\
& =\sum_{m=1}^{M} \underbrace{c_{m} e^{i\left(\omega_{x} x_{m}+\omega_{y} y_{m}\right) / c}}_{C_{m, n}^{\prime}} Z_{m}, Z_{m}=e^{i \omega_{z} z_{m} / c}
\end{aligned}
$$

where we redefine $Z_{m}$ to be unknown. By construction, the phase of the solution is uniquely determined due to the choice $\omega_{z}$, which guarantees a unique solution in the domain, hence

$$
z_{m}=\frac{\arg \left(Z_{m}\right)}{\omega_{z} / c}
$$

where $Z_{m}$ is the solution of the sensing equation (13).

\subsection{Model Mismatch and Denoising}

For the noiseless case, the system achieves exact recovery of the parameters, but in a real application the measurements are corrupted with noise. Hence, one needs to compensate for the measurement noise and model mismatches. Here we assume that the temporal measurements of the sensors, $p(\mathbf{r}, t)$ 's are corrupted with signaldepended AWGN at a certain SNR; i.e. $\tilde{p}[\mathbf{r}, l]=p[\mathbf{r}, l]+n[\mathbf{r}, l]$ and SNR $=20 \log \frac{\sum_{l=1}^{N}|p(r, l)|^{2}}{\sum_{l=1}^{N}|n(r, l)|^{2}}$.

Considering that the point absorber configuration shows joint sparsity for different frequencies, we propose to solve (10) in a joint framework such that multiple frequency samples of the measurements can be jointly used to compensate for the sensor measurements. ${ }^{11}$ Hence, the samples that are extracted according to (8) for different frequencies $\omega$ can be annihilated with the same filter defined in (9). The joint-annihilation equation will have the following form

$$
\left\{\mu_{n} \cdot R\left(a_{n}\right)\right\} * h=0, \text { for all selected } \omega
$$

where the frequency range should be chosen such that the spatial aliasing due to discrete sampling process can be controlled. 


\section{EXPERIMENTAL RESULTS}

We performed numerical experiments to validate our reconstruction algorithm. Specifically, we considered a spherical detection geometry having a radius of $8 \mathrm{~cm}$ that is typical for the imaging of breast tissue in a PAT setting. ${ }^{12}$ The speed of sound is taken as constant $c=1500 \mathrm{~m} / \mathrm{s}$ considering human breast tissue as an homogeneous medium with negligible variations in the speed of sound. ${ }^{12}$ The method achieved exact reconstruction of the absorption profile for the noiseless case assuming continuous sensor data. However, for the real application the sampling process has to be done at discrete points in space. In this section we assumed that the activated acoustic field and its normal derivative are sampled by ideal omnidirectional point detectors. Due to inevitable modal aliasing in the sampling process of a space-time signal, the frequency range of operation has to be limited properly. ${ }^{13}$ Therefore, we focused on the frequencies up to $f_{\max }=200 \mathrm{kHz}$ assuming that the temporal sampling frequency of the sensors are $1 \mathrm{MHz}$. Decomposing a wave field into a set of spherical harmonics and Bessel functions, the periodic zeros of the spherical bessel function are used to determine the necessary spatial sensor number that limits the spatial aliasing. We define the polar mode of the signal, $P$ as the maximum spatial frequency; i.e., $P=2 \pi f_{m} a x R / c$. Following the same reasoning in a sampling process with a conservative design rule, a minimum of $Q \geq(2 P)^{2}$ sensors are necessary to sample the field on the spherical surface.

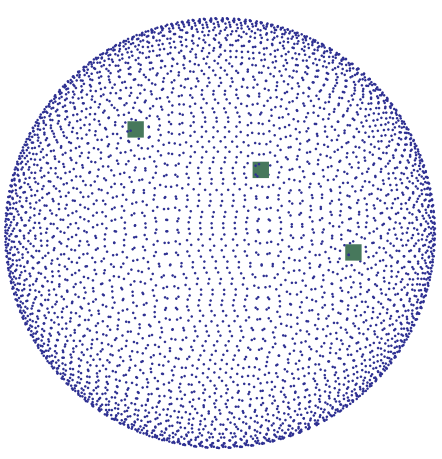

(a)

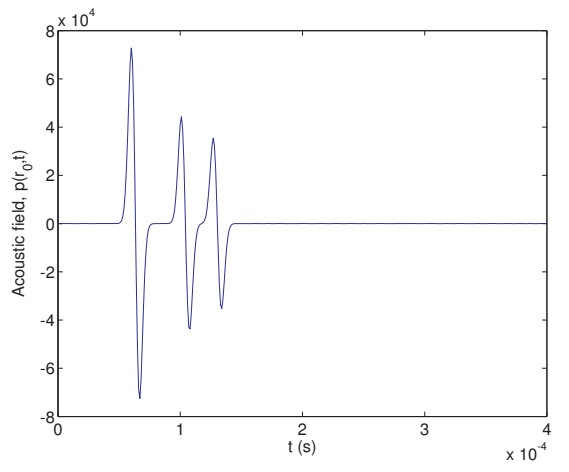

(b)

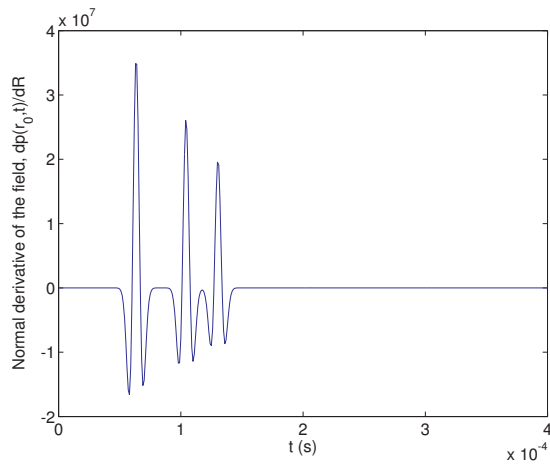

$(c)$

Figure 1: Setup for the numerical experiments (a) Spherical imaging geometry with uniformly placed sensors, (b) Sample measurement of the activated field for 3 absorbing points (c) Sample measurement of the normal derivative activated field on the surface for 3 absorbing points.

Considering the maximum spatial frequency the polar mode; i.e., $P=67$, we assumed that there are $134^{2}$ sensors uniformly positioned on the surface. The setup for the experiment is shown in Fig.1(a). We also provide a sample sensor measurement of a multiple detection problem with $M=3$ using a normalized Gaussian illumination profile $I(t)=e^{-t^{2} / 2 \sigma^{2}} / \sqrt{2 \pi \sigma^{2}}$, e.g., Fig.1 (b) and Fig.1 (c).

The performance of the proposed algorithm strongly depends on the initial step where we extract the XYprojections of the point absorbers. Therefore, we define two separate measures to evaluate each step of the method

$$
\mathrm{RMSE}=\sqrt{\frac{1}{M} \sum_{i=1}^{M}\left\|\mathbf{r}_{m}-\hat{\mathbf{r}}_{m}\right\|^{2}}
$$

where $\mathbf{r}_{m}$ is the true position, $\hat{\mathbf{r}}_{m}$ is the estimated position and we evaluate it for the XY-plane projection $\left(D_{p}\right)$ and for the full 3-D location $\left(D_{t}\right)$. In all experiments, we assumed that the weights of the absorbing points are unity and we focused on the localization accuracy of the point absorbers. In the first experiment, we compared the reconstruction error with respect to the chosen frequency of the signal. In Fig. 2 (a), the experiment was carried out for the noiseless case and considering the spatial aliasing of the sensors, we conclude that up to a $300 \mathrm{kHz}$, i.e., slightly above the frequency range of operation, it is still possible to recover the parameters. However, in Fig. 2 (b), we observe that even a for the signal measured at $20 \mathrm{~dB}$, the corruption of the noise drastically effects the performance of the method. Therefore, we used the information in the the frequency range 


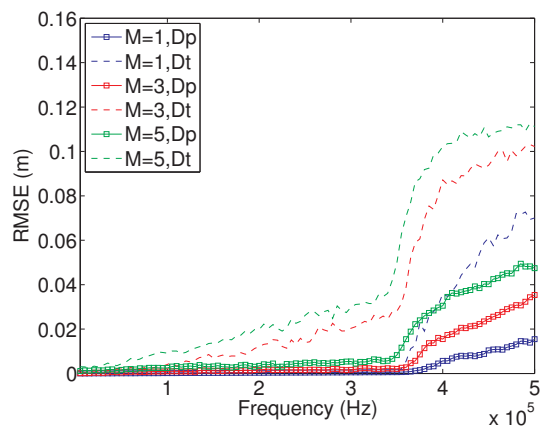

$(a)$

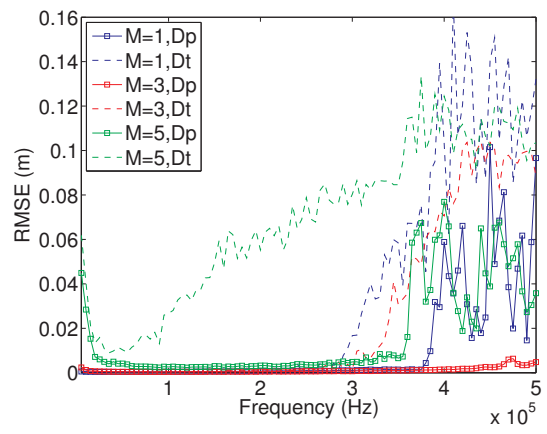

(b)

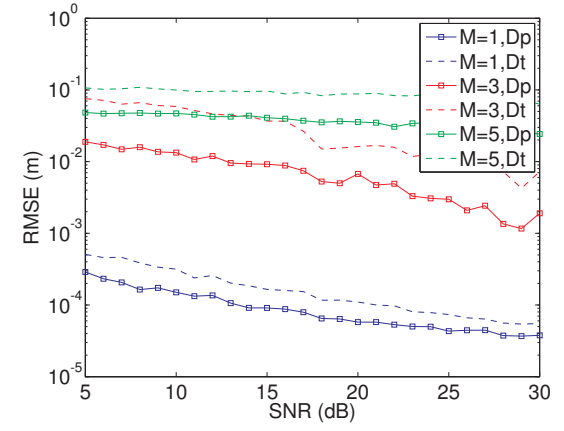

$(c)$

Figure 2: RMSE of results of 200 independent realisations (a) using the individual frequency samples of the noiseless sensor data; (b) using the individual frequency samples of the sensor data at $20 \mathrm{~dB}$; (c) using the frequency samples of the sensor data jointly with respect to different SNR levels.

of operation jointly; i.e., the frequencies between $5 \mathrm{kHz}$ and $200 \mathrm{kHz}$ with $5 \mathrm{kHz}$ steps according to (15) for the XY-plane and we picked up the data at $100 \mathrm{kHz}$ for the z-locations. For the second experiment in Fig 2 (c), we compare the localisation error with respect to the SNR level of the measurements with the proposed joint framework. Here, joint sparsity shows significant improvement upon the robustness of the estimation results even in the low SNR regime. All the experiments showed that the error in the localisation increases as the model order increases due to the interaction between the point absorbers. Considering the errors in plane, $D_{p}$ and in volume, $D_{t}$, we conclude that the accuracy of the planar projection with better localised complex sensing function (6) is more robust than the plane waves (12).

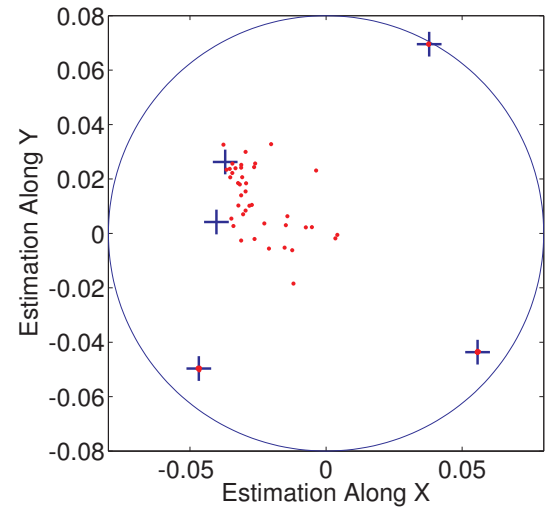

(a)

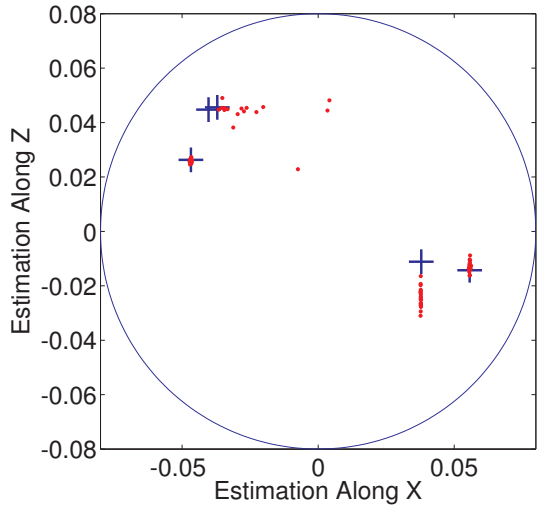

(b)

Figure 3: Scatterplot of independent realisations using the frequency samples of the sensor data jointly at $20 \mathrm{~dB}$. (a) Retrieved estimations in XY plane. (b) Retrieved estimations in XZ plane.

Finally, we provide a scatterplot for the localization of 5 point absorbers on for XY-plane and XZ-plane in Fig. 3 (a) and (b), respectively. We observe that the accuracy of the localization on XY-plane depends on the depth of the point absorbers from the measurement surface and the distance between each point absorber. We conclude that the projection onto complex plane with (6) performs robust estimation of the projected position for the point absorbers that are far enough with respect to each other. On the other hand, the accuracy on the recovery of Z locations depends highly on the estimation quality of the projected position on XY-plane and the distance from the z-axis. The plane waves (12) recovers the z-locations of the point absorbers that are far from the z-axis accurately. 


\section{CONCLUSION}

We proposed a non-iterative algorithm for PAT of point absorbers from the surface measurements of the activated field. We investigated the necessary spatial sampling rate to control the amount of spatial aliasing of the sampled data. To reconstruct accurate positioning of the point absorbers, the sensing principle requires accurate evaluation of the surface integration in (4), which necessitates dense sampling of the data. However, using the information in the frequency range of operation, we investigated the joint-sparsity model for the recovery of the parameters of the absorption profile. The experimental results revealed that the reconstruction algorithm achieves accurate results even for the low SNR regime. We have shown that the locality of the selected families of sensing functions plays a key role in the accuracy of the estimation. In our case, the sensing functions with poles on the complex plane (6) are better localized in space than the plane waves which results in increased robustness on the projection of the positions on the XY-plane. Therefore, it would be of interest to further analyse the effect of the parameters of the sensing functions to better control the locality to increase the accuracy. Another subject of future work is to extend the method for the case where full view data is not available, i.e., the sensor coverage is limited to some local neighbourhood in the half-space which is of significant practical interest. Finally, sparse models for the accurate imaging of absorption profile in PAT remains as a promising research area of further research. Moreover, we also consider the applicability and feasibility of different families of sensing functions for the imaging of sparse absorption profiles in PAT.

\section{ACKNOWLEDGMENTS}

This work was supported in part by the Swiss National Science Foundation (under the grants 205330-132808 and PP00P2-123438) and in part by the Center for Biomedical Imaging (CIBM) of the Geneva-Lausanne Universities and the EPFL and in part by the Hong Kong University Grant Council (under the RGC grant CUHK410110).

\section{REFERENCES}

1. L. V. Wang, "Multiscale photoacoustic microscopy and computed tomography," Nature photonics 3, pp. 503509, Aug. 2009.

2. L. V. Wang and S. Hu, "Photoacoustic tomography: In vivo imaging from organelles to organs," Science 335(6075), pp. 1458-1462, 2012.

3. D. Finch and S. K. Patch, "Determining a function from its mean values over a family of spheres," SIAM Journal on Mathematical Analysis 35(5), p. 1213, 2004.

4. M. Xu and L. V. Wang, "Universal back-projection algorithm for photoacoustic computed tomography," Phys. Rev. E 75, p. 059903, May 2007.

5. M. Xu, Y. Xu, and L. Wang, "Time-domain reconstruction algorithms and numerical simulations for thermoacoustic tomography in various geometries," Biomedical Engineering, IEEE Transactions on 50, pp. 1086-1099, sept. 2003.

6. Y. Xu, M. Xu, and L. V. Wang, "Exact frequency-domain reconstruction for thermoacoustic tomography: II. cylindrical geometry.," IEEE Trans. Med. Imaging 21(7), pp. 829-833, 2002.

7. G. Paltauf, J. A. Viator, S. A. Prahl, and S. L. Jacques, "Iterative reconstruction algorithm for optoacoustic imaging," The Journal of the Acoustical Society of America 112(4), pp. 1536-1544, 2002.

8. A. Buehler, A. Rosenthal, T. Jetzfellner, A. Dima, D. Razansky, and V. Ntziachristos, "Model-based optoacoustic inversions with incomplete projection data," Medical Physics 38(3), pp. 1694-1704, 2011.

9. V. Isakov, "Uniqueness and stability in multi-dimensional inverse problems," Inverse Problems 9(6), p. 579, 1993.

10. D. Kandaswamy, T. Blu, and D. Van De Ville, "Analytic sensing: Noniterative retrieval of point sources from boundary measurements," SIAM Journal on Scientific Computing 31(4), pp. 3179-3194, 2009.

11. Z. Dogan, I. Jovanovic, T. Blu, and D. Van De Ville, "3D reconstruction of wave-propagated point sources from boundary measurements using joint sparsity and finite rate of innovation," in Biomedical Imaging (ISBI), 2012 9th IEEE International Symposium on, pp. 1575 -1578, may 2012.

12. M. Xu and L. Wang, "Photoacoustic imaging in biomedicine," Rev. Sci. Instrum. 77(4), p. 041101, 2006.

13. H. Teutsch and W. Kellermann, "Acoustic source detection and localization based on wavefield decomposition using circular microphone arrays," Journal of the Acoustical Society of America 120, pp. 2724-2736, NOV 2006. 